\title{
TWO NEW TOPOLOGICAL CARDINAL INEQUALITIES
}

\author{
SUN SHU-HAO \\ (Communicated by Dennis Burke)
}

\begin{abstract}
In this paper, two new cardinal inequalities are obtained. The first one is a common generalization of inequalities of Hajnal-Juhasz and Sapirovskii; the second one generalizes the inequality of Arhangelskir.
\end{abstract}

It is well known that Hajnal-Juhasz's inequality [3], "For $X \in T_{2},|X| \leq$ $2^{c(X) \chi(X) "}$ and Sapirovskii's inequality [2], "For $X \in T_{3},|X| \leq \pi \chi(X)^{c(X) \psi(X) "}$ are still two of the best cardinal inequalities. In other words, they have not been improved so far [2].

In the first part of this paper, we will establish a stronger inequality, "For $X \in T_{2}$, $|X| \leq \pi \chi(X)^{c(X) S \psi(X)}$ " which is the common generalization of the two inequalities above. Here, we define a collection $\mathscr{U}$ of open sets of $X$ to be a strong pseudo-local base at $p$ if $\{p\}=\bigcap\{U: U \in \mathscr{U}\}=\bigcap\{\bar{U}: U \in \mathscr{U}\}$. Then we define:

$$
\begin{aligned}
& S \psi(p, X)=\min \{|\mathscr{U}|: \mathscr{U} \text { is a strong pseudo-local base at } p\} \cdot \omega, \\
& S \psi(X)=\sup \{S \psi(p, X): p \in X\} .
\end{aligned}
$$

It is immediate that $\psi(X)=S \psi(X)$ for $T_{3}$ spaces and that $\chi(X) \geq S \psi(X)$ for $T_{2}$ spaces. The latter inequality can be strict; for example, consider the subspace $N \cup\{p\}$ of $\beta N$, where $p \in \beta N \backslash N$.

For each space $X$ it is obvious that $\pi \chi(X) \leq \chi(X)<2^{c(X) \chi(X)}$. Thus

$$
\pi \chi(X)^{c(X) S \psi(X)} \leq\left(2^{c(X) \chi(X)}\right)^{c(X) S \psi(X)} \leq\left(2^{c(X) \chi(X)}\right)^{c(X) \chi(X)}=2^{c(X) \chi(X)} .
$$

Hence our new inequality is at least as strong as that of Hajnal and Juhasz. Later we give an example to show that the above inequality can be strict. Because $\psi(X)=S \psi(X)$ when $X$ is $T_{3}$, it follows that our inequality also generalizes that of Sapirovskii mentioned above.

Our proofs make use of the Pol-Sapirovskii technique. We shall use the notation and terminology for cardinal functions employed in [3]. For the convenience of the reader, we repeat some of the definitions contained in this paper.

First, let $|A|$ denote the cardinality of $A$; let $\lambda, k$ be infinite cardinals and let $\omega$ denote the smallest infinite ordinal and the smallest infinite cardinal. The successor of $k$ will be denoted by $k^{+}$.

DEFINITIONS. Let $X$ be a topological space and $\mathscr{U}$ be a collection of nonempty open sets of $X$. Let $p \in X$; then $\mathscr{U}$ is a local $\pi$-base at $p$ if for each neighbourhood

Received by the editors January 27, 1987 and, in revised form, August 11, 1987.

1980 Mathematics Subject Classification (1985 Revision). Primary 54A25.

Key words and phrases. Cardinal inequality, strong pseudo-character, quasi-dense. 
$R$ of $p$, there exists a $V \in \mathscr{U}$ such that $V \subseteq R$. We define

$$
\begin{aligned}
\pi \chi(p, X) & =\min \{|\mathscr{U}|: \mathscr{U} \text { is a local } \pi \text {-base for } p\} \cdot \omega ; \\
\pi \chi(X) & =\sup \{\pi \chi(p, X): p \in X\} ; \\
\chi(p, X) & =\min \{|\mathscr{U}|: \mathscr{U} \text { is a local base for } p\} \cdot \omega ; \\
\chi(X) & =\sup \{\chi(p, X): p \in X\} .
\end{aligned}
$$

A collection $\mathscr{U}$ of open sets in $X$ is said to be a pseudo-local base for $p$, if $\{p\}=\bigcap\{U: U \in \mathscr{U}\}$. Now we define

$$
\begin{aligned}
\psi(p, X) & =\min \{|\mathscr{U}|: \mathscr{U} \text { is a pseudo-local base for } p\} \cdot \omega ; \\
\psi(X) & =\sup \{\psi(p, X): p \in X\} .
\end{aligned}
$$

A pairwise disjoint collection of nonempty open sets of $X$ is said to be a cellular family. We define $c(X)=\sup \{|\mathscr{U}|: \mathscr{U}$ is a cellular family in $X\}$.

The notation $[A]^{\leq k}$ denotes the set $\{B: B \subseteq A,|B| \leq k\}$.

THEOREM 1. For $X \in T_{2},|X| \leq \pi \chi(X)^{c(X) S \psi(X)}$.

Proof. Let $\pi \chi(X)=\lambda$ and $c(X) S \psi(X)=k$; for each $p$ in $X$, let $\mathscr{U}_{p}$ be a $\pi$-base for $p$ such that $\left|\mathscr{U}_{p}\right| \leq \lambda$.

Construct a sequence $\left\{A_{\alpha}: \alpha<k^{+}\right\}$of subsets of $X$ and a sequence $\left\{\mathscr{U}_{\alpha}: 0<\right.$ $\left.\alpha<k^{+}\right\}$of open collections in $X$ such that

(1) $\left|A_{\alpha}\right| \leq \lambda^{k}, 0 \leq \alpha<k^{+}$

(2) $\mathscr{U}_{\alpha}=\left\{V: \exists p \in \bigcup_{\beta<\alpha} A_{\beta}\right.$ such that $\left.V \in \mathscr{U}_{p}\right\}, 0<\alpha<k^{+}$;

(3) For each $r<k$, if $\mathscr{V}_{r} \in\left[\mathscr{U}_{\alpha}\right] \leq k$ and $W=\bigcup_{r<k} \overline{\bigcup_{\mathscr{V}}} \neq X$, then $A_{\alpha} \backslash W \neq \varnothing$.

The construction is by transfinite induction. Let $0<\alpha<k^{+}$and assume that $\left\{A_{\beta}: \beta<\alpha\right\}$ has already been constructed. Then $\mathscr{U}_{\alpha}$ is defined by (2), i.e., we put $\mathscr{U}_{\alpha}=\left\{V: \exists p \in \bigcup_{\beta<\alpha} A_{\beta}, V \in \mathscr{U}_{p}\right\}$. It follows that $\left|\mathscr{U}_{\alpha}\right| \leq \lambda^{k}$. If $\left\{\mathscr{V}_{r}\right\}_{r<k} \in\left[\left[\mathscr{U}_{\alpha}\right]^{\leq k}\right]^{\leq k}$ and $W=\bigcup_{r<k} \overline{\mathscr{V}_{r}} \neq X$, then we can choose one point of $X \backslash W$. Let $S_{\alpha}$ be the set of points chosen in this way. Since $\left|\left[\left[\mathscr{U}_{\alpha}\right]^{\leq k}\right] \leq k\right| \leq \lambda^{k}$, one has $\left|S_{\alpha}\right| \leq \lambda^{k}$. Define $A_{\alpha}$ to be the set $S_{\alpha} \cup\left(\bigcup_{\beta<\alpha} A_{\beta}\right)$. Then $A_{\alpha}$ satisfies (1), and (3) is also satisfied if $\beta \leq \alpha$. This completes the construction.

Now let $S=\bigcup_{\alpha<k^{+}} \bar{A}_{\alpha}$; then $|S| \leq k^{+} \lambda^{k}=\lambda^{k}$. The proof is complete if $S=X$. Suppose not and let $p \in X \backslash S$; since $S \psi(X) \leq k$, there exist open neighbourhoods $\left\{U_{\alpha}(p)\right\}_{\alpha<k}$ of $p$ such that $\{p\}=\bigcap_{\alpha<k}\left\{\overline{U_{\alpha}(p)}\right\}$. For each $\alpha<k$, let $V_{\alpha}=X \backslash \overline{U_{\alpha}(p)}$, then $p \notin \bar{V}_{\alpha}$, and $\bigcup_{\alpha<k} V_{\alpha}=X \backslash\{p\} \supset S$.

For each $\alpha<k$, and for each $q \in\left(V_{\alpha} \cap S\right) \subseteq V_{\alpha}$, there exists a $V_{q} \in \mathscr{U}_{q}$ such that $V_{q} \subseteq V_{\alpha}$. Let $\mathscr{W}_{\alpha}=\left\{V: V \in \mathscr{U}_{q}, q \in V_{\alpha} \cap S, V \subseteq V_{\alpha}\right\} ;$ then $G_{\alpha}=\bigcup \mathscr{W}_{\alpha} \subseteq V_{\alpha}$, and it is easy to check that $V_{\alpha} \cap S \subseteq G_{\alpha}$. As $c(X) \leq k$, there exists a $\mathscr{V}_{\alpha} \in\left[\mathscr{W}_{\alpha}\right] \leq k$ such that $\overline{\cup \mathscr{V}_{\alpha}}=\overline{G_{\alpha}} \supseteq V_{\alpha} \cap S$, and $p \notin \overline{\cup \mathscr{V}_{\alpha}}$. Let $W=\bigcup_{\alpha<k} \overline{\cup \mathscr{V}_{\alpha}}$ then $p \notin W$.

Since $\mid\left\{V: \exists \alpha<k\right.$ such that $\left.V \in \mathscr{V}_{\alpha}\right\} \mid \leq k k=k<k^{+}$, there is an $\alpha_{0}<k^{+}$ such that $\mathscr{V}_{\alpha} \in\left[\mathscr{U}_{\alpha_{0}}\right] \leq k$ for each $\alpha<k$. Hence, by (3), one has $A_{\alpha_{0}+1} \backslash W \neq \varnothing$. But $W \supseteq \bigcup_{\alpha<k}\left(V_{\alpha} \cap S\right)=S$. This is a contradiction.

Corollary 1 (SAPIROvSKII). For $X \in T_{3},|X| \leq \pi \chi(X)^{c(X) \psi(X)}$.

Proof. It is clear that $\psi(X)=S \psi(X)$ for $X \in T_{3}$. 
COROllaRY 2 (HAJNAL-JUHASZ). For $X \in T_{2},|X| \leq 2^{c(X) \chi(X)}$.

ProOF. By this theorem, $|X| \leq \pi \chi(X)^{c(X) S \psi(X)} \leq 2^{\pi \chi(X) c(X) S \psi(X)} \leq 2^{c(X) \chi(X)}$.

REMARK 1. In this theorem, we omitted the condition "regularity". This improvement on Sapirovskii's theorem is not trivial, as there exist $T_{2}$ spaces for which Sapirovskii's inequality fails. For example, the Katetov $H$-closed extension of the positive integers, denoted $k N$, is such a space as $|k N|=2^{c}, \pi \chi(k N)=\omega$, and $\psi(k N)=\omega$.

REMARK 2. Our theorem represents a considerable improvement on the theorem of Hajnal and Juhasz quoted above, as the gap between $\pi \chi(X)^{c(X) S \psi(X)}$ and $2^{c(X) \chi(X)}$ can be large.

To illustrate this, let us consider the Cantor cube $D^{c}=\prod_{s \in S} D_{s}$, where $D_{s}=$ $D, D$ denotes the two-point discrete space, for every $s \in S$, and $|S|=c$. It contains a dense countable subspace $X$ such that $\chi(X)=c[\mathbf{1}]$ and it is clear that $\psi(X)=c(X)=|X|=\omega$. By the regularity of $X$, one has $S \psi(X)=\omega$. Note that $\pi \chi(X) \leq \chi(X)=c=2^{\omega}$. So $\pi \chi(X)^{c(X) S \psi(X)} \leq\left(2^{\omega}\right)^{\omega \omega}=2^{\omega}=c$. But $2^{c(X) \chi(X)}=2^{c}$.

We also note that if $p \in \beta N \backslash N$, the space $N \cup\{p\}$ illustrates the same point.

Now we turn to the second result of this paper. We shall establish a theorem which strengthens Arhangelskii's theorem, "For $X \in T_{2},|X| \leq 2^{t(X) L(X) \psi(X) " ~(s e e ~}$ [2, Remark 4.6]), where $t(X)$ and $L(X)$ denote the tightness and Lindelöf degree of $X$, respectively.

Let $k$ be a cardinal number. We define a subset $A$ of $X$ with $|A| \leq 2^{k}$ to be $k$-quasi-dense if for each open cover $\mathscr{U}$ of $X$ there exist a $\mathscr{V} \in[\mathscr{U}] \leq k$ and a $B \in[A]^{\leq k}$ such that $(\bigcup \mathscr{V}) \cup \bar{B}=X$; and let us write $q L(X)=\min \{k:$ there is a $k$-quasi-dense subset $A\}$. (The notion of $q L(X)$ was first considered by Liu Xiao-Shi.) Clearly, $q L(X)$ is less than both the density $d(X)$ and Lindelöf degree $L(X)$. We also can prove that $q L(X) \leq s(X)$, where

$$
s(X) \quad(=\sup \{|D|: D \subseteq X, D \text { is discrete }\} \omega)
$$

denotes the spread of $X$. In fact, by virtue of the theorem of Sapirovskii, "Let $X \in T_{2}$, let $s(X) \leq k$. Then there is a subset $S$ of $X$ with $|S| \leq 2^{k}$ such that $X=\bigcup\{\bar{A}: A \subseteq S,|A| \leq k\}$ ", we have only to show that the subset $S$ is a $k$-quasidense subset of $X$. Let $\mathscr{U}$ be an open cover of $X$. By virtue of another theorem of Sapirovskii which asserts that if $\mathscr{U}$ is an open cover of $X$ with $s(X) \leq k$, then there is a subset $B$ of $X$ with $|B| \leq k$ and a subcollection $\mathscr{V}$ of $\mathscr{U}$ with $|\mathscr{V}| \leq k$ such that $X=\bar{B} \cup(\cup \mathscr{V})$ [2, Proposition 4.8], we can easily find a subset $A$ of $S$ with $|A| \leq k$ such that $X=\bar{A} \cup(\bigcup \mathscr{V})$. In fact, for each $b \in B$, there is a subset $A(b)$ of $S$ with $|A(b)| \leq k$ such that $b \in \overline{A(b)}$. Let $A=\bigcup\{A(b): b \in B\}$; then $A$ is as required.

LEMMA. Let $X$ be a space with $t(X) S \psi(X) \leq k$. Then for each subset $A$ of $X$ with $|A| \leq 2^{k}$, we have $|\bar{A}| \leq 2^{k}$.

Proof. Let $x \in \bar{A}$; since $S \psi(X) \leq k$, there exists a strong pseudo-local base $\left\{U_{\alpha}(X): \alpha<k\right\}$ at $x$. Thus, $\{x\}=\bigcap_{\alpha<k} \overline{U_{\alpha}(x) \cap A}$. Since $t(X) \leq k$, for each $\alpha<k$, there exists $A_{\alpha} \subseteq U_{\alpha}(x) \cap A \subseteq A$ such that $\left|A_{\alpha}\right| \leq k$ and $x \in \bar{A}_{\alpha}$. Hence $\{x\}=\bigcap_{\alpha<k} \bar{A}_{\alpha}$ and $\left\{A_{\alpha}\right\}_{\alpha<k} \in[[A] \leq k] \leq k$. Therefore $|\bar{A}| \leq|[[A] \leq k] \leq k|=2^{k}$. 
REMARK. The above lemma generalizes Pospisil's inequality, which states that if $X \in T_{2}$, then $|X| \leq d(X)^{\chi(X)}[2$, Theorem 4.4].

THEOREM 2. For $X \in T_{2},|X| \leq 2^{q L(X) t(X) S \psi(X)}$.

Proof. Let $q L(X) t(X) S \psi(X)=k$; and let $A$ be a $k$-quasi-dense subset of $X$. For each $x \in X$, let $\mathscr{V}_{x}$ denote a family of open neighbourhoods $\left\{U_{\alpha}(x)\right\}_{\alpha<k}$ such that $\{x\}=\bigcap_{\alpha<k} \overline{U_{\alpha}(x)}$ and $\left|\mathscr{V}_{x}\right| \leq k$. Using the above lemma, we construct an increasing sequence $\left\{H_{\alpha}: 0 \leq \alpha<k^{+}\right\}$of closed sets of $X$ and a sequence $\left\{\mathscr{V}_{\alpha}: 0<\alpha<k^{+}\right\}$of open collections in $X$ such that:

(1) $\left|H_{\alpha}\right| \leq 2^{k}, 0 \leq \alpha<k^{+}$;

(2) $\mathscr{V}_{\alpha}=\left\{V: V \in \mathscr{V}_{p}, p \in \bigcup_{\beta<\alpha} H_{\beta}\right\}, 0<\alpha<k^{+}$;

(3) if $W$ is the union of $\leq k$ elements of $\mathscr{V}_{\alpha}, B \in[A] \leq k$ and $X \backslash(W \cup \bar{B}) \neq \varnothing$, then $H_{\alpha} \backslash(W \cup \bar{B}) \neq \varnothing$.

Now let $H=\bigcup_{\alpha<k^{+}} H_{\alpha}$; as $t(X) \leq k$ and $H_{\alpha}$ is closed for each $\alpha<k^{+}, H$ is closed. We will show that $H \cup \bar{A}=X$, from which it follows that $|X| \leq|H|+|\bar{A}| \leq$ $2^{k}$.

Let $q \in X \backslash H$. For each $p \in H$, choose a $V_{p} \in \mathscr{V}_{p}$ such that $q \notin \bar{V}_{p}$. Now $\bigcup\left\{V_{p}: p \in H\right\} \supseteq H$. Since $q L(X) \leq k$, there exist a $\mathscr{V} \in\left[\left\{V_{p}: p \in H\right\}\right] \leq k$ and a $B \in[A] \leq k$ such that $H \subseteq(\bigcup \mathscr{V}) \cup \bar{B}$. If $q \in \bar{B} \subseteq \bar{A}$, then the conclusion holds. If $q \notin \bar{B}$, then $q \notin(\bigcup \mathscr{V}) \cup \bar{B}$, i.e., $(\bigcup \mathscr{V}) \cup \bar{B} \neq X$. Hence we can find a $\beta<k^{+}$ with $\mathscr{V} \in\left[\mathscr{V}_{\beta}\right]^{\leq k}$ since $|\mathscr{V}| \leq k$ and $H=\bigcup_{\alpha<k^{+}} H_{\alpha}$. So $H_{\beta} \backslash((\bigcup \mathscr{V}) \cup \bar{B}) \neq \varnothing$, which contradicts with the fact that $H \subseteq(\bigcup \mathscr{V}) \cup \bar{B}$.

COROllary. For $X \in T_{2}$, we have $|X| \leq 2^{L(X) t(X) \psi(X)}$.

Proof. It is easily checked that $S \psi(X) \leq L(X) \psi(X)$, so

$$
L(X) \psi(X)=L(X) S \psi(X) .
$$

The conclusion now follows from the theorem above.

EXAMPLE. Let $X$ be the Niemytzki plane. Then $d(X)=q L(X)=\chi(X)=$ $t(X)=S \psi(X)=\omega$, but $L(X) t(X) \psi(X) \geq L(X)>\omega$.

REMARK. In Theorem $2, S \psi(X)$ cannot be replaced by $\psi(X)$ since there exists a Hausdorff space $X$ of cardinality $2^{c}$ (namely the Katetov $H$-closed extension of $N$ ) that contains a countable dense subset $A$ consisting of isolated points of $X$ such that the subspace $X \backslash A$ is discrete. It is easy to check $q L(X)=d(X)=\psi(X)=t(X)$, but $|X|>c=2^{q L(X) t(X) \psi(X)}$.

ACKNOWLEDGEMENT. The author would like to thank the referee for many valuable suggestions and very careful corrections.

\section{REFERENCES}

1. R. Engelking, General topology, PWN, Warszawa, 1977.

2. R. E. Hodel, Cardinal functions. I, Handbook of Set-Theoretic Topology (K. Kunen and J. E. Vaughan, eds.), North-Holland, 1984, pp. 1-61.

3. I. Juhasz, Cardinal functions in topology-ten years later, Math. Centrum, Amsterdam, 1980.

4. B. Sapirovskii, Canonical sets and character, density and weight in compact spaces, Soviet Math. Dokl. 15 (1974), 1282-1287.

Department of MAthematics, Shanghai Institute of Mechanical ENGineERing, Shanghai, Peoples Republic of China 